archives

of thermodynamics

Vol. 38(2017), No. 3, 77-99

DOI: $10.1515 /$ aoter-2017-0017

\title{
The effect of pulsed laser radiation on a thermoviscoelastic semi-infinite solid under two-temperature theory
}

\author{
MOHAMED I. OTHMAN ${ }^{a, b}$, \\ AHMED E.E. ABOUELREGAL ${ }^{c, d} *$ \\ a Department of Mathematics, Faculty of Science, Zagazig University, \\ P.O. Box 44519 Zagazig, Egypt. \\ ${ }^{b}$ Department of Mathematics, Faculty of Science, Taif University, 888, \\ Taif, Saudi Arabia \\ $c$ Department of Mathematics, Faculty of Science, Mansoura University, \\ Mansoura 35516, Egypt \\ $d$ Department of Mathematics, College of Science and Arts, University of \\ Aljouf, El-Qurayat, Saudi Arabia
}

\begin{abstract}
The purpose of this paper is to study the thermoviscoelastic interactions in a homogeneous, isotropic semi-infinite solid under twotemperature theory with heat source. The Kelvin-Voigt model of linear viscoelasticity which describes the viscoelastic nature of the material is used. The bounding plane surface of the medium is subjected to a non-Gaussian laser pulse. The generalized thermoelasticity theory with dual phase lags model is used to solve this problem. Laplace transform technique is used to obtain the general solution for a suitable set of boundary conditions. Some comparisons have been shown in figures to estimate the effects of the phase lags, viscosity, temperature discrepancy, laser-pulse and the laser intensity parameters on all the studied fields. A comparison was also made with the results obtained in the case of one temperature thermoelasticity theory.
\end{abstract}

Keywords: Generalized thermo-visco-elasticity; Two-temperature; Non-Gaussian laser pulse; Laser intensity; phase lags; Kelvin-Voigt model

${ }^{*}$ Corresponding Author. Email: ahabogal@mans.edu.eg 


\section{Nomenclature}

$\begin{array}{ll}b & - \text { temperature discrepancy factor } \\ c_{1}=\sqrt{\left(\lambda_{e}+2 \mu_{e}\right) / \rho} & - \text { longitudinal wave speed } \\ C_{E} & - \text { specific heat at uniform strain } \\ e_{i j} & - \text { linear strain tensor } \\ k=K / \rho C_{E} & - \text { thermal diffusivity } \\ K & - \text { thermal conductivity } \\ L_{0} & - \text { laser intensity } \\ q_{i} & - \text { components of heat flux vector } \\ Q & - \text { intensity of heat source } \\ R_{a} & - \text { surface reflectivity } \\ t_{p} & - \text { characteristic time of the laser pulse } \\ t_{0} & - \text { mechanical relaxation time due to the viscosity } \\ T_{0} & - \text { environment temperature } \\ T & - \text { absolute temperature of the medium } \\ u, w & - \text { displacement components } \\ (x, y, x) & - \text { Cartesian coordinates system }\end{array}$

\section{Greek symbols}

$\begin{array}{lll}\alpha_{1}, \alpha_{2} & - & \text { thermoviscoelastic relaxation times } \\ \alpha_{t} & - & \text { thermal expansion coefficient } \\ \delta_{i j} & - & \text { Kronecker delta function } \\ \delta_{1} & - & \text { absorption depth of heating energy } \\ \eta=\rho C_{E} / K & - & \text { thermal viscosity } \\ \theta=T-T_{0} & - & \text { temperature change } \\ \lambda_{e}, \mu_{e} & - & \text { Lameñs constant } \\ \rho & - & \text { material density } \\ \sigma_{i j} & - & \text { mechanical stress components } \\ \tau_{q} & - & \text { phase lag of the heat flux } \\ \tau_{\theta} & - & \text { phase lag of gradient of temperature } \\ \varphi & - & \text { conductive temperature }\end{array}$

\section{Introduction}

The theory of thermoelasticity deals with the effects of mechanical and thermal disturbances on elastic body. Biot has introduced a theory of coupled thermoelasticity to overcome the first shortcoming [1]. The governing equations for this theory are coupled, eliminating the first paradox of the classical theory. However, this theory shares the second shortcoming since the heat equation for the coupled theory is based on Fourier's law of heat conduction and is also parabolic. Henceforth, two styles of generalized theories of thermoelasticity presented by Lord and Shulman [2] and Green and Lindsay [3], which admit the finite speed of the thermal signal, have 
been the center of interest of active research during the last three decades. The third generalization to the coupled theory is known as the dual phase lag (DPL) thermoelasticity, proposed by Tzou [4], in which Fourier's law is replaced by an approximation to a modification of Fourier law with two different translations for the heat flux and the temperature gradient. These theories remove the paradox of the infinite speed of heat propagation that is inherent in the conventional coupled dynamical theory of thermoelasticity, which was introduced by Biot [1]. In the generalized theories, a modified heat conduction law, which includes both the heat flux and its time derivative, replaced the conventional Fourier law.

Chen and Gurtin [5] and Chen et al. [6,7] have formulated a theory of heat conduction in deformable bodies, which depends upon two distinct temperatures, the conductive and thermodynamic temperatures. For timeindependent situations, the difference between these two temperatures is proportional to the heat supply, and in the absence of any heat supply, the two temperatures are identical. For time-dependent problems, however, and for wave propagation problems in particular, the two temperatures are in general different regardless of the presence of heat supply. The two temperatures and the strain are found to have representations in the form of a traveling wave plus a response, which occurs instantaneously throughout the body [8]. Warren and Chen have investigated the wave propagation in the two-temperature theory of thermoelasticity (2TT) [9]. Youssef has extended this theory in the context of the generalized theory of thermoelasticity [10].

Pulsed laser irradiation is employed over a wide spectrum of materials processing applications, including surface hardening, alloying, curing, synthesis of compound and superconductor films. An extensive review of pulsed laser processing of semiconductors is given in [11]. Very rapid thermal processes, under the action of an ultrashort laser pulse, are interesting from the standpoint of thermoelasticity, since they require an analysis of the coupled temperature and deformation fields as in [12]. This mechanism has attracted considerable attention due to extensive application of pulsed laser technologies in material processing and nondestructive detection and characterization as in [13]. The so-called ultrashort lasers are those with pulse duration ranging from nanoseconds to femtoseconds in general. The non-Fourier effect of heat conduction takes into account the effect of mean free time in the energy carrier's collision process, which can eliminate this contradiction. Wang and $\mathrm{Xu}$ studied the stress wave induced by nano-, 
pico-, and femto-second laser pulses in a semi-infinite solid [13]. The solution takes into account the non-Fourier effect in heat conduction and the coupling effect between temperature and strain rate. It is known that characteristic elastic waveforms are generated when a pulsed laser irradiates a metal surface. McDonald studied the importance of thermal diffusion to the thermoelastic wave generation [14]. Allam and Abouelregal have discussed the thermoelastic waves induced by pulsed laser and varying heat of nonhomogeneous microscale beam resonators [15]. Othman et al. studied the effect of rotation on a semiconducting medium with two-temperature under L-S theory. The effect of internal friction on the propagation of plane waves in an elastic medium may also be considered owing to the fact that dissipation accompanies vibrations in solid media due to the conversion of elastic energy to heat energy [16].

The effect of internal friction on the propagation of plane waves in an elastic medium may also be considered owing to the fact that dissipation accompanies vibrations in solid media due to the conversion of elastic energy to heat energy.

The viscoelastic nature of a medium has special significance in wave propagation in a solid medium. The Kelvin-Voigt model is one of the macroscopic mechanical models often used to describe the viscoelastic behavior of a material. This model represents the delayed elastic response subjected to stress when the deformation is time dependent but recoverable. The dynamical interaction of thermal and mechanical fields in solids has a number of significant practical applications in modern aeronautics, astronautics, nuclear reactors, and high energy particle accelerators, for example. Abd-alla and Abo-Dahab $[17,18]$ investigated the reflection of the generalized magneto-thermo-viscoelastic plane waves. Mukhopadhyay studied the thermal relaxation effects and compared the various theories of generalized thermoelasticity for thermo-viscoelastic interactions in an infinite viscoelastic solid of Kelvin-Voigt type with a spherical cavity [19]. Mukhopadhyay and Bera investigated the effect of distributed instantaneous continuous heat sources in an infinite conducting magneto-thermoviscoelastic solid with relaxation time [20]. Ezzat et al. introduced the state-space approach for the two-dimensional model of generalized thermoviscoelasticity with two relaxation times [21].

Othman and Song studied the effect of rotation on plane waves of the generalized electromagneto-thermo-viscoelasticity with two relaxation times [22]. Othman and Fekry explain the effect of magnetic field on gen- 
eralized thermo-viscoelastic diffusion medium with voids [23].

The objective of the present investigation is to determine the components of displacement, stress, conductive temperature, thermodynamic temperature distributions and strain in an isotropic homogeneous viscoelastic half space. The governing equations are derived in the context of twotemperature generalized thermoelasticity with phase lags. The bounding plane surface is heated by a non-Gaussian laser beam. An exact solution of the problem is first obtained in the Laplace transform space. The inversion of the Laplace transform will be computed numerically by using a method based on Fourier expansion technique. The derived expressions are computed numerically for copper and the results are presented in graphical form. The effects of the two-temperature parameter, the laser-pulse, the laser intensity and viscosity parameters are estimated.

\section{Governing equation of two-temperature thermoelasticity with phase lags}

The basic governing equations of motion and heat conduction, in the context of the generalized theory of thermoelasticity, in the absence of body forces are given in [19].

The equations of motion without body forces take the forms

$$
\mu^{\prime} u_{i, j j}+\left(\lambda^{\prime}+\mu^{\prime}\right) u_{j, i j}-\beta^{\prime} \theta_{, i}=\rho \frac{\partial^{2} u_{i}}{\partial t^{2}} .
$$

The constitutive equations take the forms

$$
\sigma_{i j}=2 \mu^{\prime} e_{i j}+\delta_{i j}\left(\lambda^{\prime} e_{k k}-\beta^{\prime} \theta\right),
$$

where $\sigma_{i j}$ is the stress tensor and $\delta_{i j}$ is Kronecker's delta function, $u_{i}$ are the components of the displacement vector, $t$ is the time, $e_{i j}$ is the strain, $\theta=T-T_{0}, T$ is the absolute temperature of the medium, $T_{0}$ is the reference uniform temperature of the body chosen such $\left|\theta / T_{0}\right|<<1, \rho$ is the density. In the above equations, a prime symbol refers to spatial derivative, a dot refers to temporal derivative and summation convention is used.

The parameters $\lambda^{\prime}, \mu^{\prime}$ and $\beta^{\prime}$ are defined as [19]

$$
\begin{gathered}
\lambda^{\prime}=\lambda_{e}\left(1+\alpha_{1} \frac{\partial}{\partial t}\right), \quad \mu^{\prime}=\mu_{e}\left(1+\alpha_{2} \frac{\partial}{\partial t}\right), \quad \beta^{\prime}=\beta_{e}\left(1+\beta \frac{\partial}{\partial t}\right), \\
\beta_{e}=\left(3 \lambda_{e}+2 \mu_{e}\right) \alpha_{t}, \quad \beta=\left(3 \lambda_{e} \alpha_{1}+2 \mu_{e} \alpha_{2}\right) \frac{\alpha_{t}}{\beta_{e}},
\end{gathered}
$$


where $\lambda_{e}, \mu_{e}$ being Lamé constants, $\alpha_{1}, \alpha_{2}$ are the thermoviscoelastic relaxation times, and $\alpha_{t}$ is the coefficients of linear thermal expansion.

Fourier's law states that the heat flow is proportional to the temperature gradient. The constant of proportionality depends, among other things, on a material parameter known as the thermal conductivity of the material. For heat conduction to occur there must be temperature differences between neighboring points. The classical thermoelasticity is based on the principles of the classical theory of heat conductivity, specifically on the classical Fourier law, which relates the heat flux vector $\mathbf{q}_{i}$ to the temperature gradient as follows:

$$
\mathbf{q}_{i}=-K \theta_{, i},
$$

where $K$ is the thermal conductivity of a solid and $\theta=T-T_{0}, T$ is the absolute temperature of the medium, $T_{0}$ is the reference uniform temperature of the body chosen such $\left|\theta / T_{0}\right|<<1$, which together with the energy equation yields the heat conduction equation or the parabolic heat conduction equation and is diffusive with the notion of infinite speed of propagation of thermal disturbances

$$
\rho C_{E} \frac{\partial \theta}{\partial t}+\beta^{\prime} T_{0} \frac{\partial}{\partial t} u_{i, i}=-\mathrm{q}_{i, i}+Q,
$$

where $C_{E}$ is the specific heat and $Q$ is the intensity of heat source.

The modified form of classical thermoelasticity model is given by Tzou theory in which the Fourier law is replaced by an approximation of the equation [22]

$$
\mathbf{q}_{i}\left(x, t+\tau_{q}\right)=-K \theta_{, i}\left(x, t+\tau_{\theta}\right),
$$

where $\tau_{\theta}$ is the phase lag of the heat flux, and $\tau_{q}$ is phase lag of gradient of temperature. The above equation may be approximated by [22]

$$
\left(1+\tau_{q} \frac{\partial}{\partial t}\right) \mathbf{q}_{i}=-K\left(1+\tau_{\theta} \frac{\partial}{\partial t}\right) \theta_{, i} .
$$

In classification of real material into simple and nonsimple materials Chen and Gurtin [5] have proposed a theory of nonsimple materials for which thermodynamics and conductive temperatures are not identical unlike simple materials for which they are identical. This theory was further extended to deformable bodies by Chen et al. [6,7]. They have shown that the equation of heat conduction for such materials contains an additional term involving the time derivative of the Laplacian of the conductive temperature; the equation of motion also includes terms involving the space derivatives 
of the Lapalcian of conductive temperature, Considering isotropy and the linearity, for such materials, they have shown that the two temperatures are related by the relation [7]

$$
\varphi-\theta=b \varphi_{, i i}
$$

where $\varphi$ is the conductive temperature, $\theta$ is thermodynamic temperature, and $b>0$ is the temperature discrepancy factor.

Now, we assume a new generalized heat conduction equation of the form

$$
\left(1+\tau_{q} \frac{\partial}{\partial t}\right) \mathbf{q}_{i}=-K\left(1+\tau_{\theta} \frac{\partial}{\partial t}\right) \varphi_{, i},
$$

taking the divergence of both sides of the above equation, one gets

$$
\left(1+\tau_{q} \frac{\partial}{\partial t}\right) q_{i, i}=-K\left(1+\tau_{\theta} \frac{\partial}{\partial t}\right) \varphi_{, i i} .
$$

The generalized equation of heat conduction with two temperatures in the case of nonsimple medium using Eqs. (2) and (8) takes the form [22]

$$
K\left(1+\tau_{\theta} \frac{\partial}{\partial t}\right) \varphi=\left(1+\tau_{q} \frac{\partial}{\partial t}\right)\left[\rho C_{E} \frac{\partial \theta}{\partial t}+\beta^{\prime} T_{0} \frac{\partial}{\partial t} u_{i, i}-Q\right]
$$

The above equation may be considered as the generalized heat conduction equation in isotropic, thermoelastic solids with two temperatures. The key element that sets the two-temperature thermoelasticity theory apart from the classical theory is the material parameter $b$. Specifically, in the limit as $b \rightarrow 0, \varphi \rightarrow \theta$, the classical theory (one-temperature generalized thermoelasticity theory $1 \mathrm{TT}$ ) is recovered.

\section{Statement of the problem}

We consider a homogenous isotropic thermoviscoelastic half space occupying a half-space $x \geq 0$ and initially undisturbed and at uniform temperature $T_{0}$. The Kelvin-Voigt model of linear viscoelasticity which describes the viscoelastic nature of the material has been employed to study the problem. So, the initial conditions for all field variables are homogeneous. This halfspace is irradiated uniformly the bounding plane $(x=0)$ by a laser pulse with the non-Gaussian temporal profile. The system is initially quiescent where all the state functions are depending only on the variable $x$ and the 
time $t$.

The displacement components for a one-dimensional medium have the forms $u_{x}=u(x, t)$ and $u_{y}=u_{z}=0$. The relation between the strain and displacement can be expressed as $e=e_{x x}=\partial u / \partial x$. The constitutive equation will be

$$
\sigma_{x x}=\sigma=\left(\lambda^{\prime}+2 \mu^{\prime}\right) e-\beta^{\prime} \theta .
$$

The equation of motion takes the form

$$
\left(\lambda^{\prime}+2 \mu^{\prime}\right) \frac{\partial^{2} u}{\partial x^{2}}-\beta^{\prime} \frac{\partial \theta}{\partial x}=\rho \ddot{u}_{i}
$$

where the double dot refers to second derivative with respect to time or may be written in the form

$$
\frac{\partial^{2} \sigma}{\partial x^{2}}=\rho \frac{\partial^{2} e}{\partial t^{2}}
$$

The relation between the heat conduction and the thermodynamic heat takes the form

$$
\varphi-\theta=b \frac{\partial^{2} \varphi}{\partial x^{2}} .
$$

The heat conduction equation (10) is given by

$$
K\left(1+\tau_{\theta} \frac{\partial}{\partial t}\right) \frac{\partial^{2} \varphi}{\partial x^{2}}=\left(1+\tau_{q} \frac{\partial}{\partial t}\right)\left[\rho C_{E} \frac{\partial}{\partial t}\left(\varphi-b \frac{\partial^{2} \varphi}{\partial x^{2}}\right)+\beta^{\prime} T_{0} \frac{\partial e}{\partial t}-Q\right]
$$

Let's introduce the following nondimensional parameters:

$$
\begin{gathered}
\left(x^{\prime}, u^{\prime}\right)=c_{1} \eta(x, u), \quad\left(t^{\prime}, \tau_{q}^{\prime}, \tau_{\theta}^{\prime}, \beta_{1}^{\prime}, \alpha_{2}^{\prime}, \alpha_{1}^{\prime}\right)=c_{1}^{2} \eta\left(t^{\prime}, \tau_{q}^{\prime}, \tau_{\theta}^{\prime}, \beta_{1}^{\prime}, \alpha_{2}^{\prime}, \alpha_{1}^{\prime}\right), \\
\theta^{\prime}=\frac{\beta_{e} \theta}{\rho c_{1}^{2}}, \quad \varphi^{\prime}=\frac{\beta_{e} \varphi}{\rho c_{1}^{2}}, \quad \sigma^{\prime}=\frac{\sigma}{\rho c_{1}^{2}}, \quad Q^{\prime}=\frac{Q}{K c_{1}^{2} \eta^{2} T_{0}}
\end{gathered}
$$

where $c_{1}=\sqrt{\left(\lambda_{e}+2 \mu_{e}\right) / \rho}$ is the longitudinal wave speed and $\eta=\rho C_{E} / K$ is the thermal viscosity. Then, Eqs. (12) and (14)-(16) can be transformed into the dimensionless forms

$$
\begin{gathered}
\sigma=\left(1+\delta_{1} \frac{\partial}{\partial t}\right) e-\left(1+\beta \frac{\partial}{\partial t}\right) \theta, \\
\frac{\partial^{2} \sigma}{\partial x^{2}}=\frac{\partial^{2} e}{\partial t^{2}},
\end{gathered}
$$




$$
\begin{gathered}
\varphi-\theta=\omega \frac{\partial^{2} \varphi}{\partial x^{2}} \\
\left(1+\tau_{\theta} \frac{\partial}{\partial t}\right) \frac{\partial^{2} \varphi}{\partial x^{2}}=\left(1+\tau_{q} \frac{\partial}{\partial t}\right)\left[\frac{\partial \theta}{\partial t}+\varepsilon\left(1+\beta \frac{\partial}{\partial t}\right) \frac{\partial e}{\partial t}-Q\right],
\end{gathered}
$$

where $\varepsilon=\beta_{e}^{2} T_{0} / \rho^{2} C_{E} c_{1}^{2}, \omega=b c_{1}^{2} \eta^{2}, \delta=\frac{c_{2}^{2}}{c_{1}^{2}}, \quad c_{2}^{2}=\frac{\lambda_{e} \alpha_{1}+2 \mu_{e} \alpha_{2}}{\rho}$.

Now, let us consider the medium is uniformly heated by a laser pulse with the non-Gaussian form temporal profile [25] as

$$
I(t)=\frac{L_{0} t}{t_{p}^{2}} e^{-t / t_{p}}
$$

where $t_{p}$ is a characteristic time (measured by picoseconds) of the laserpulse (the time duration of a laser pulse), $L_{0}$ is the laser intensity which is defined as the total energy carried by a laser pulse per unit area of the laser beam. The conduction heat transfer in the medium can be modeled as a one dimensional problem with the energy source, $Q(x, t)$, near the surface:

$$
Q(x, t)=\frac{R_{a}}{\delta_{1}} e^{(x-h / 2) / \delta_{1}} I(t)=\frac{R_{a} L_{0}}{\delta_{1} t_{p}^{2}} t e^{(x-h / 2) / \delta_{1}-t / t_{p}},
$$

where $\delta_{1}$ is the absorption depth of heating energy and $R_{a}$ is the surface reflectivity [25]. Note that the laser pulse may lie on the surface of the medium $(x=0)$. In this case, the energy source takes the form

$$
Q(t)=\frac{R_{a} L_{0}}{\delta_{1} t_{p}^{2}} t e^{-h /\left(2 \delta_{1}\right)-t / t_{p}} .
$$

\section{Initial and boundary conditions}

The problem is solved under proper initial and boundary conditions:

$$
\begin{gathered}
\theta(x, t)=\varphi(x, t)=u(x, t)=0 \quad \text { at } \quad t=0, \\
\frac{\partial \theta(x, t)}{\partial t}=\frac{\partial \varphi(x, t)}{\partial t}=\frac{\partial u(x, t)}{\partial t}=0 \quad \text { at } \quad t=0 .
\end{gathered}
$$

The thermal and mechanical boundary conditions on the bounding plane, $x=0$, of the assumed half-space are given as follows: 
- Thermal boundary condition:

The boundary plane $x=0$ is subjected to a thermal shock as follows:

$$
\theta(0, t)=\theta_{0} H(t)
$$

where $H(t)$ is called the Heaviside unit step function and $\theta_{0}$ is a constant.

- Mechanical boundary condition:

The boundary plane $x=0$ is considered to be friction free,

$$
\sigma(0, t)=0 .
$$

\section{Solution of the problem in the Laplace transform domain}

Applying Laplace transform with respect to variable $t$ for Eqs. (18)-(21), one can get the system of differential equations in the transformed domain as follows:

$$
\begin{gathered}
\bar{\sigma}=\left(1+\delta_{1} s\right) \bar{e}-(1+\beta s) \bar{\theta}, \\
\frac{\mathrm{d}^{2} \bar{\sigma}}{\mathrm{d} x^{2}}=s^{2} \bar{e}, \\
\bar{\theta}=\bar{\varphi}-\omega \frac{d^{2} \bar{\varphi}}{d x^{2}}, \\
\frac{d^{2} \bar{\varphi}}{d x^{2}}=\alpha_{0}[\bar{\theta}+\varepsilon(1+\beta s) \bar{e}]-\bar{G}(s),
\end{gathered}
$$

where

$$
\bar{G}(s)=\frac{R_{a} L_{0} e^{-h / 2 \delta_{1}}}{t_{p}^{2} c_{1} \delta_{1}\left(1+\tau_{\theta} s\right)}\left[\frac{t_{p} \tau_{q}}{1+s t_{p}}+\frac{t_{p}^{2}-t_{p} \tau_{q}}{\left(1+s t_{p}\right)^{2}}\right], \quad \alpha_{0}=\frac{s\left(1+\tau_{q} s\right)}{1+\tau_{\theta} s} .
$$

Eliminating $\bar{\theta}$ and $\bar{e}$ from Eqs. (28)-(31), one obtains

$$
\left[\frac{d^{4}}{d x^{4}}-A \frac{d^{2}}{d x^{2}}+B\right] \bar{\varphi}=q_{2} \bar{G}(s),
$$

where

$$
A=\frac{\alpha_{0} \omega+q_{1} \alpha_{0}+q_{2} q_{3}}{\alpha_{0} \varepsilon \omega(1+\beta s)+q_{1} q_{3}}, \quad B=\frac{q_{2} \alpha_{0}}{\alpha_{0} \varepsilon \omega(1+\beta s)+q_{1} q_{3}},
$$




$$
q_{1}=\frac{1+\delta_{1} s}{(1+\beta s)}, \quad q_{2}=\frac{s^{2}}{(1+\beta s)}, \quad q_{3}=1+\alpha_{1} \omega .
$$

The solution of Eq. (33) takes the following form:

$$
\bar{\varphi}=A_{1} e^{-m_{1} x}+A_{2} e^{-m_{2} x}+\bar{F}_{1}(s),
$$

where $A_{1}$ and $A_{2}$ are parameters of $s$.

The solution of $\bar{\varphi}$ is used in Eq. (30) to get the solution of $\bar{\theta}$

$$
\bar{\theta}=\left(1-\beta m_{1}^{2}\right) A_{1} e^{-m_{1} x}+\left(1-\beta m_{2}^{2}\right) A_{2} e^{-m_{2} x}+\bar{F}_{1}(s) .
$$

The above two solutions are used in Eq. (31) to get the solution of $\bar{e}$ as

$$
\begin{gathered}
\bar{e}=\frac{\bar{G}(s)}{\varepsilon \alpha_{0}(1+\beta s)}+\left[\frac{q_{3}}{\varepsilon \alpha_{0}(1+\beta s)} D^{2}-\frac{\alpha_{1}}{\varepsilon \alpha_{0}(1+\beta s)}\right] \bar{\varphi} \\
=\bar{F}_{2}(s)+\Omega_{1} A_{1} e^{-m_{1} x}+\Omega_{2} A_{2} e^{-m_{2} x}
\end{gathered}
$$

where

$$
\bar{F}_{1}(s)=q_{2} \bar{G}(s) / B, \quad \bar{F}_{2}(s)=\left[\frac{B-\alpha_{0} q_{2}}{B \varepsilon \alpha_{0}(1+\beta s)}\right] \bar{G}(s), \Omega_{i}=\frac{q_{3} m_{i}^{2}-\alpha_{0}}{\varepsilon \alpha_{0}(1+\beta s)},
$$$$
i=1,2 \text {. }
$$

and substituting Eqs. (36) and (37) into Eq. (28), one obtains

$$
\bar{\sigma}=\bar{F}(s)+\Gamma_{1} A_{1} e^{-m_{1} x}+\Gamma_{2} A_{2} e^{-m_{2} x},
$$

where: $\bar{F}(s)=\bar{F}_{2}(s)\left(1+\delta_{1} s\right)-(1+\beta s) \bar{F}_{1}(s)$,

$$
\Gamma_{i}=\Omega_{1}\left(1+\delta_{1} s\right)-(1+\beta s)\left(1-\beta m_{i}^{2}\right), i=1,2 .
$$

From Eq. (14) and by using the non-dimensional variables and placeLaplace transforms, one obtains the displacement in the following form:

$$
\bar{u}=\frac{1}{s^{2}} \frac{\partial \bar{\sigma}}{\partial x}=-m_{1} \Gamma_{1} A_{1} e^{-m_{1} x}-m_{2} \Gamma_{2} A_{2} e^{-m_{2} x} .
$$

In addition, the thermal and mechanical boundary conditions in the Laplace domain $\bar{\theta}(0, s)=\theta_{0} / s$ and $\bar{\sigma}(0, s)=0$ with the aid of Eqs. (36) and (39), gives

$$
\begin{gathered}
A_{1}=\frac{s \bar{F}(s)\left(\beta m_{2}^{2}-1\right)+\bar{F}(s)\left(s \bar{F}_{1}(s)-\theta_{0}\right)}{s\left[\Gamma_{1}-\Gamma_{2}+\beta\left(\Gamma_{2} m_{1}^{2}-\Gamma_{1} m_{2}^{2}\right)\right]}, \\
A_{2}=-\frac{s \bar{F}(s)\left(\beta m_{1}^{2}-1\right)+\bar{F}(s)\left(s \bar{F}_{1}(s)-\theta_{0}\right)}{s\left[\Gamma_{1}-\Gamma_{2}+\beta\left(\Gamma_{2} m_{1}^{2}-\Gamma_{1} m_{2}^{2}\right)\right]} .
\end{gathered}
$$

This completes the solution in the Laplace transform domain. 


\section{Special cases}

\subsection{Generalized thermoelastic theory with two-temperature (2TTE)}

Neglecting viscous effect by taking $\alpha_{1}=\alpha_{2}=0$, we obtain the expressions for the displacement components, stresses and temperature field in the generalized magneto-thermoelastic heat conduction equation with diffusion.

\subsection{Generalized thermoelastic theory with one-temperature (1TTE)}

Neglecting viscous effect by taking $\alpha_{1}=\alpha_{2}=0$ and $\omega \rightarrow 0$, we obtain the expressions for the displacement components, stresses and temperature field in the generalized thermoelastic with theory one-temperature.

\subsection{Generalized thermoviscoelastic theory with one-temperature (1TTVE)}

The generalized thermoviscoelastic theory with one-temperature can be deduced by setting the two-temperature parameter $\omega \rightarrow 0$.

\section{Numerical inversion of the Laplace transform}

In order to determine the conductive and thermal temperature, displacement and stress distributions in the time domain, we adopt a numerical inversion method based on a Fourier series expansion [26]. In this method, the inverse $f(t)$ of the Laplace transform $\bar{f}(s)$ is approximated by the relation

$$
f(t)=\frac{e^{\zeta t}}{t_{1}}\left\{\frac{1}{2} \bar{f}(\zeta)+R e\left[\sum_{k=0}^{N} \bar{f}\left(\zeta+\frac{i k \pi}{t_{1}}\right) e^{i k \pi t / t_{1}}\right]\right\}, \quad 0 \leq t \leq t_{1}
$$

where Re is the real part and $i$ is imaginary number unit, and $N$ is a sufficiently large integer representing the number of terms in the truncated infinite Fourier series. It must be chosen such that

$$
e^{\zeta t} e^{i N \pi t / t_{1}} \operatorname{Re}\left[\bar{f}\left(\zeta+\frac{i N \pi}{t_{1}}\right)\right] \leq \varepsilon_{1},
$$


where $\varepsilon_{1}$ is a persecuted small positive number that corresponds to the degree of accuracy to be achieved. The parameter $\zeta$ is a positive free parameter that must be greater than the real parts of all singularities of $\bar{f}(s)$. The optimal choice of $\zeta$ was obtained according to the criteria described in $[26]$.

\section{Numerical results and discussion}

With the aim to illustrate the theoretical results obtained in the preceding sections, we now present some numerical results. Material chosen for this purpose is copper, featuring the following the physical data:

$$
\begin{gathered}
K=368 \mathrm{~N} / \mathrm{Ks}, \quad \alpha_{t}=1.78 \times 10^{-5} \mathrm{~K}^{-1}, \quad C_{E}=383.1 \mathrm{~m}^{2} / \mathrm{K}, \\
\rho=8954 \mathrm{~kg} / \mathrm{m}^{3}, \quad \lambda_{e}=7.76 \times 10^{10} \mathrm{~N} / \mathrm{m}^{2}, \\
\mu_{e}=3.86 \times 10^{10} \mathrm{~N} / \mathrm{m}^{2}, \quad T_{0}=293 \mathrm{~K}, \alpha_{1}=0.06 \mathrm{~s}, \alpha_{2}=0.09 \mathrm{~s} .
\end{gathered}
$$

The computations were carried out for a wide range of $x(0 \leq x \leq 1)$ at small value of time $t=0.15$. The physical quantities are plotted in Figs. $1-$ 15. For all numerical calculations one takes $\delta_{1}=0.01, \tau_{0}=0.02, R_{a}=0.5$, and $h=0.1$. The field quantities such as the conductive temperature, dynamical temperature, stress, strain, and displacement distributions depend not only on the time $t$ and space coordinate $x$, but also depend on the twotemperature parameter $\omega$ and the laser intensity $L_{0}$. The laser intensity $L_{0}$ is assumed to be of the form $L_{0}=\xi \times 10^{11} \mathrm{~J} / \mathrm{m}^{2}$ where $\xi$ is the laser intensity parameter. Using these values, nondimensional field variables have been evaluated and results are presented in the form of graphs. In order to analyze the effects of different parameters, such as, heat source, viscosity, two-temperature on displacement, temperatures and stress, we have considered their graphical representations into three categories.

In the first case, Figs. 1-4 plot the displacement $u$, thermodynamic temperature $\theta$, conductive temperature $\varphi$ and the stress $\sigma$ distributions with different values of the two-temperature parameter $\omega$ to stand for the effect of this parameter on all the studied fields. The value of $\omega=0$ indicates the old situation (one temperature thermoviscoelasticity theory 1TTVE) while $\omega=0.02$ or 0.04 indicates the two-temperature thermoviscoelasticity theory (2TTVE). In this case one takes $\tau_{q}=0.2, \tau_{\theta}=0.1$, $\xi=1$, and $t_{p}=2$. The wave-amplitude of the displacement $u$ decreases as $\omega$ increases. For $x>0.1$, the thermodynamic temperature $\theta$ increases as $\omega$ 


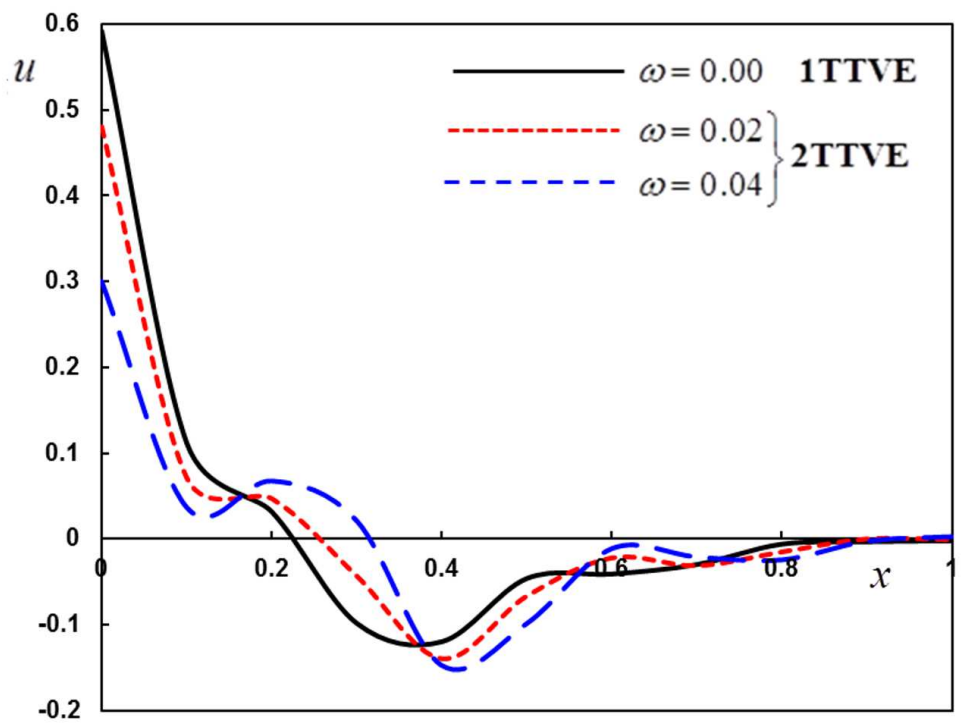

Figure 1: Dependence of displacement $u$ on the two temperature parameter $\omega$ along distance $x$.

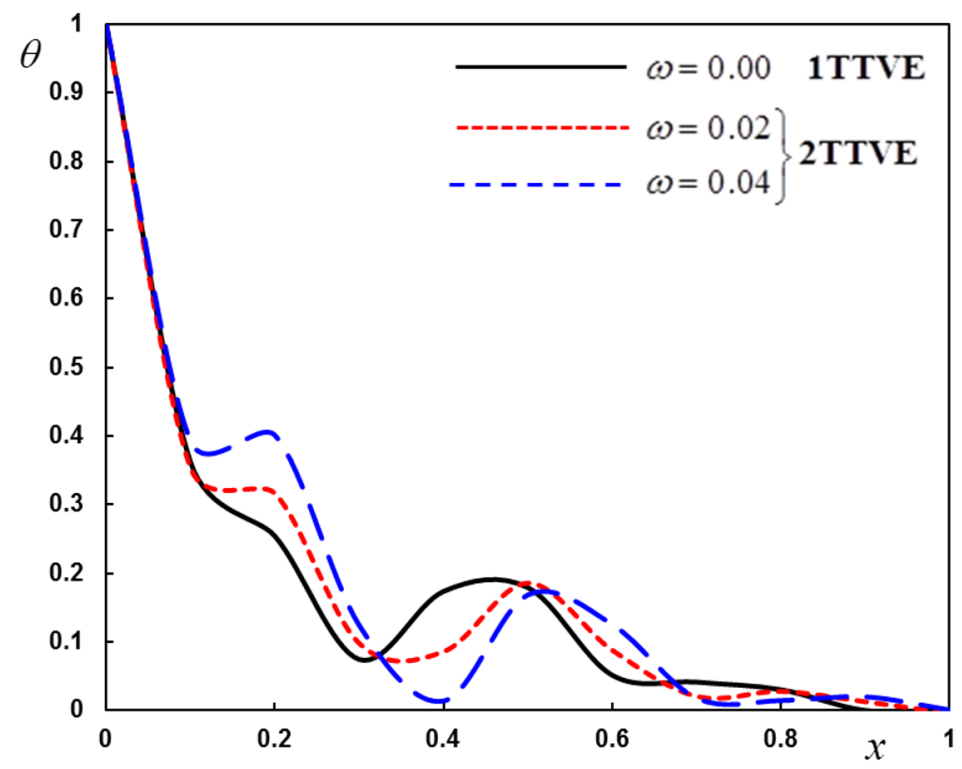

Figure 2: Dependence of thermodynamical temperature $\theta$ on the two temperature parameter $\omega$ along distance $x$. 


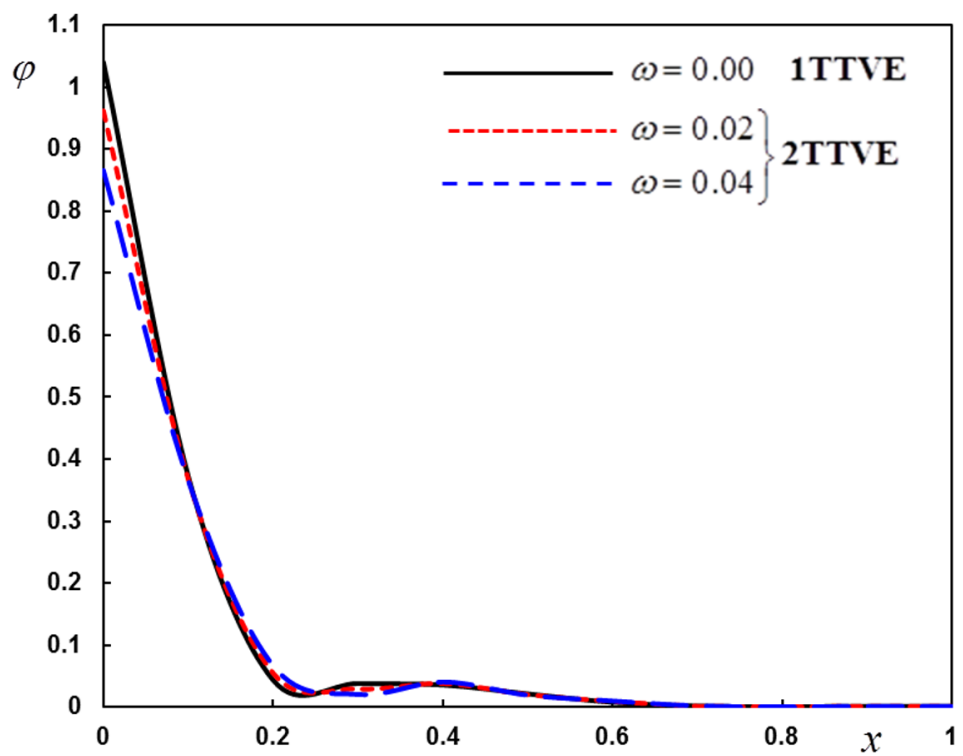

Figure 3: Dependence of conductive temperature $\varphi$ on the two temperature parameter $\omega$ along distance $x$.

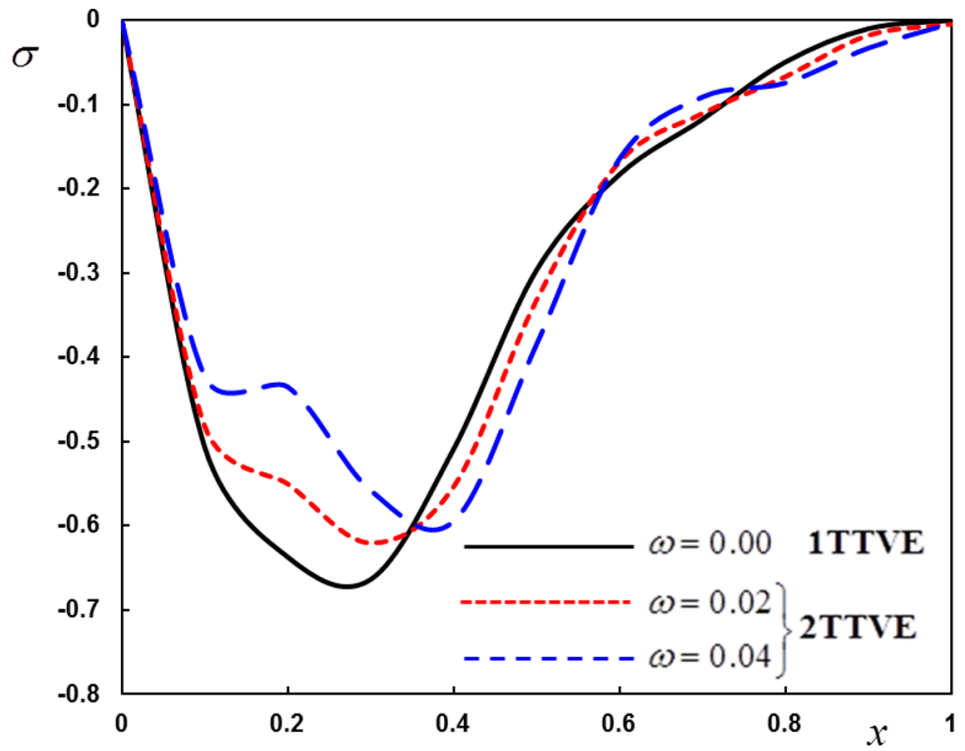

Figure 4: Dependence of the thermal stress $\sigma$ on the two temperature parameter $\omega$ along distance $x$ 
increases. Also, as $\omega$ increases the conductive temperature $\varphi$ decreases in the interval $0<x<0.1$. In most positions, the stress $\sigma$ increases as $\omega$ increases in the intervals $0<x<0.37$ and $0.6<x<0.1$ while it decreases in the interval $0.37<x<0.6$ and decreases in the interval $0.28<x<1$.

This shows the difference between the one temperature viscothermoelasticity model with phase lags 1TTVE (when $\omega=0$ ) and the two-temperature generalized viscothermoelasticity 2TTVE (when $\omega=0.02$ or 0.04 ). The figures show that this parameter has a significant effect on all the fields. The waves reach the steady state depending on the value of temperature discrepancy $\omega$. Also these figures indicate that, the two-temperature generalized theory of thermoelasticity describes the behavior of the particles of an elastic body more realistically than the one-temperature theory of generalized thermoelasticity. From Fig. 4 , the stress at $x=0$ is zero as shown, which agrees with the boundary condition prescribed. This coincided with the mechanical boundary condition that the medium surface is friction free. In general, the amplitude of the wave of the displacement $u$ is decreasing along the distance $x$. The thermodynamically $\theta$ and the conductive $\varphi$ temperatures are directly decreasing along the distance $x$.

In the second case, Figs. 5-8 plot field quantities for different values of the laser intensity parameter $\xi$ to stand for the effect of this parameter. It is found that this parameter has significant effects with fixed values of $\omega=0.02$ and $t_{p}=2$. It is observed that the nature of variations of all field variables for laser intensity parameter $\xi$ is significantly different. All fields increase when the value of $\xi$ increases.

In the last case, different values of the dual phase lag (DPL) of the heat flux and the temperature gradient $\tau_{q}$ and $\tau_{\theta}$, respectively, are considered. The graphs in Figs. 9-12 represent the curves predicted by three different theories of thermoelasticity obtained as special cases of the present DPL model. The computations were performed for various values of the parameters $\tau_{q}$ and $\tau_{\theta}$ to obtain the coupled theory (CTE) $\left(\tau_{q}=\tau_{\theta}=0\right)$, the Lord-Shulman (LS) theory $\left(\tau_{\theta}=0, \tau_{q}=0.2\right)$, and the generalized theory of thermoelasticity proposed by Tzou (DPL) $\left(\tau_{q}=0.2, \tau_{\theta}=0.1\right)$.

It can be observed that the PLs parameters have a great effect on the distribution of field quantities. The mechanical distributions indicate that the wave propagates as a wave with finite velocity in medium. The values in classical theory of thermoelasticity (CTE model) are different compared to those of other theories. The fact that in generalized thermoelasticity theories (DPL and LS), the waves propagate with finite speeds is evident 


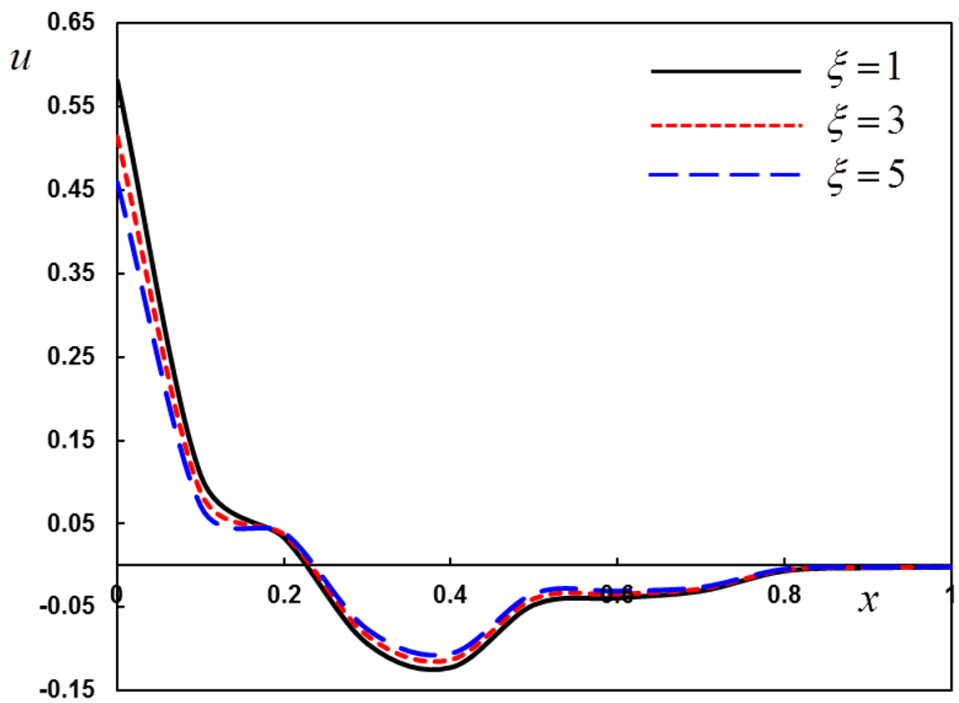

Figure 5: Dependence of displacement $u$ on the laser intensity $\xi$ along distance $x$.

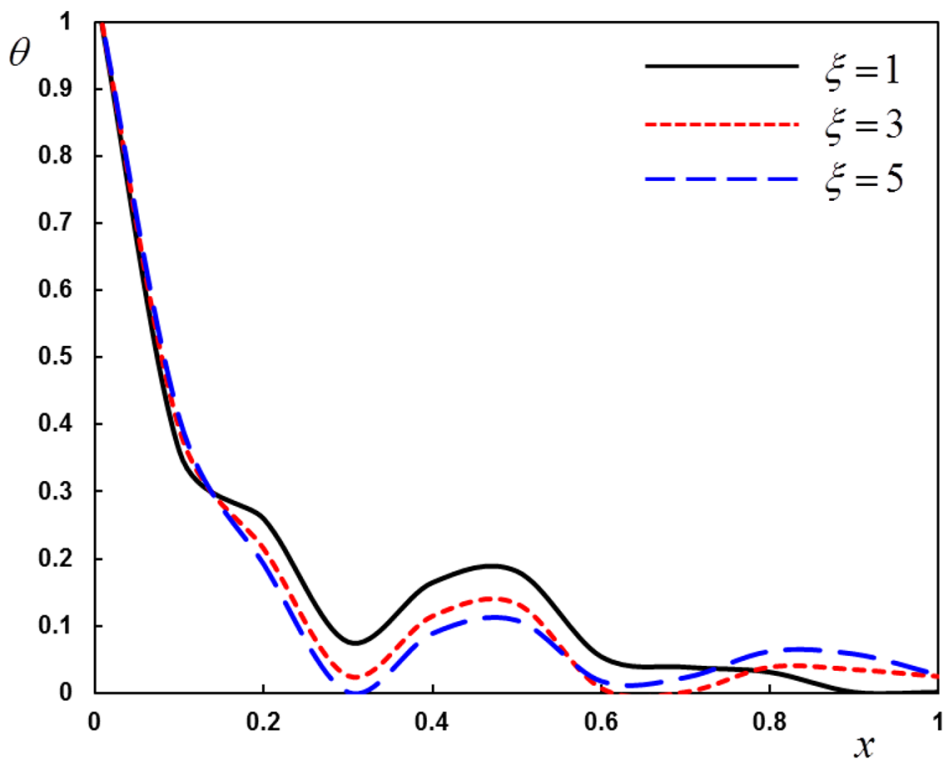

Figure 6: Dependence of thermodynamical temperature $\theta$ on the laser intensity $\xi$ along distance $x$. 


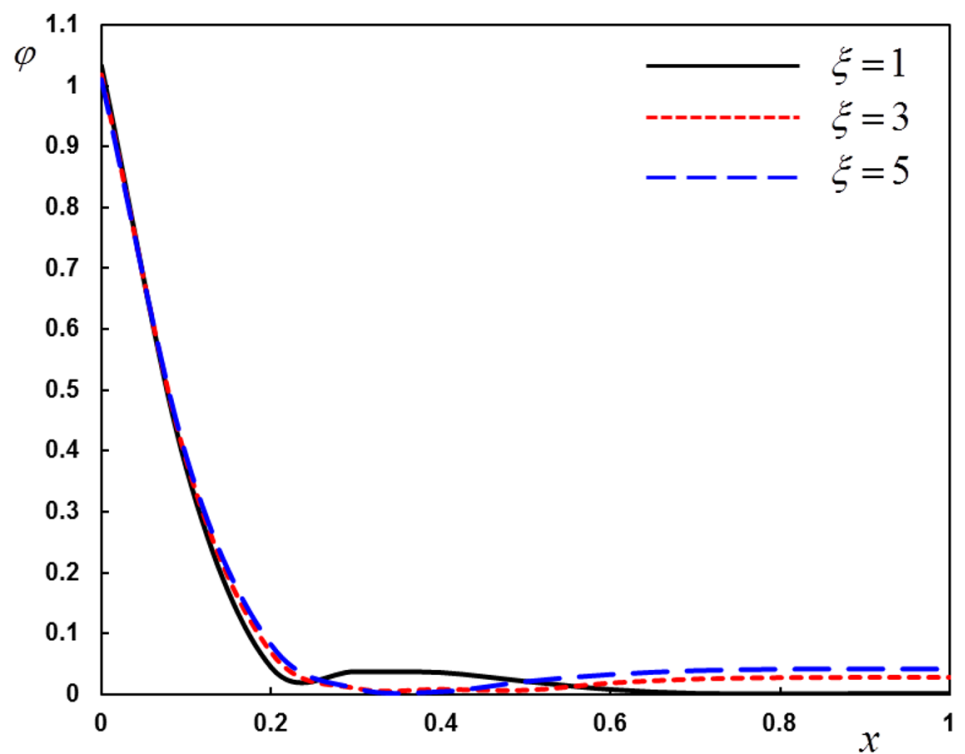

Figure 7: Dependence of conductive temperature $\varphi$ on the time of the laser intensity $\xi$ along distance $x$.

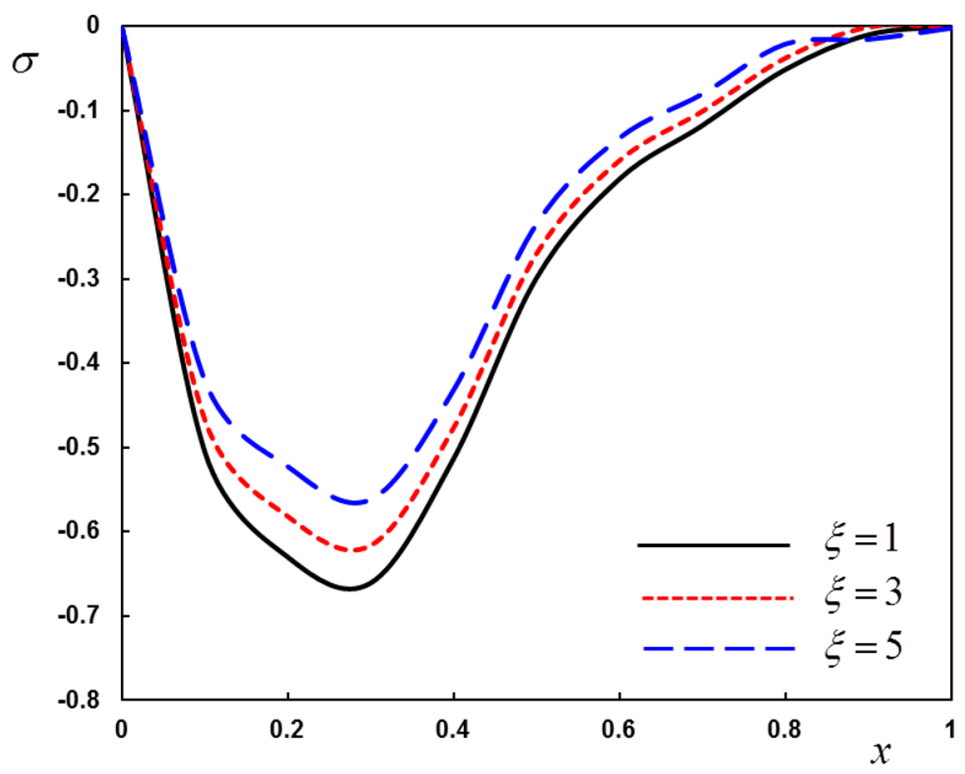

Figure 8: Dependence of the thermal stress $\sigma$ on the laser intensity $\xi$ along distance $x$. 
in all these figures. The behavior of the three theories is generally quite similar.

\section{Concluding remarks}

In this paper, the theory of two-temperature thermoviscoelasticity with phase lags was constructed and applied to a specific problem of a semiinfinite solid. The effect of the laser intensity and phase lags of the heat flux and phase lage of gradient of temperatureand $\tau_{q}$ as well as the twotemperature parameter on the field variables has been investigated.

The results concluded from the above analysis can be summarized as follows:

1. The presence of phase lags parameters play a significant role in all physical quantities.

2. It is seen that the values of all field variables are significantly dependent on the two-temperature parameter.

3. According to the theory of thermoviscoelasticity with two-temperatures, we have to construct a new classification for materials where this parameter becomes a new indicator of its ability to conduct heat under the effect of thermoelastic properties.

4. It is also observed that the theories of coupled thermoelasticity and generalized thermoelasticity with one relaxation time can be obtained as limited cases.

5. From our results, we can consider the theory of two-temperature generalized thermoviscoelasticity as an improvement on studying elastic materials.

6. The properties of a body depend largely on the laser intensity of applied source. Therefore, the presence of the non-Gaussian laser pulse in the current model is of significance. 


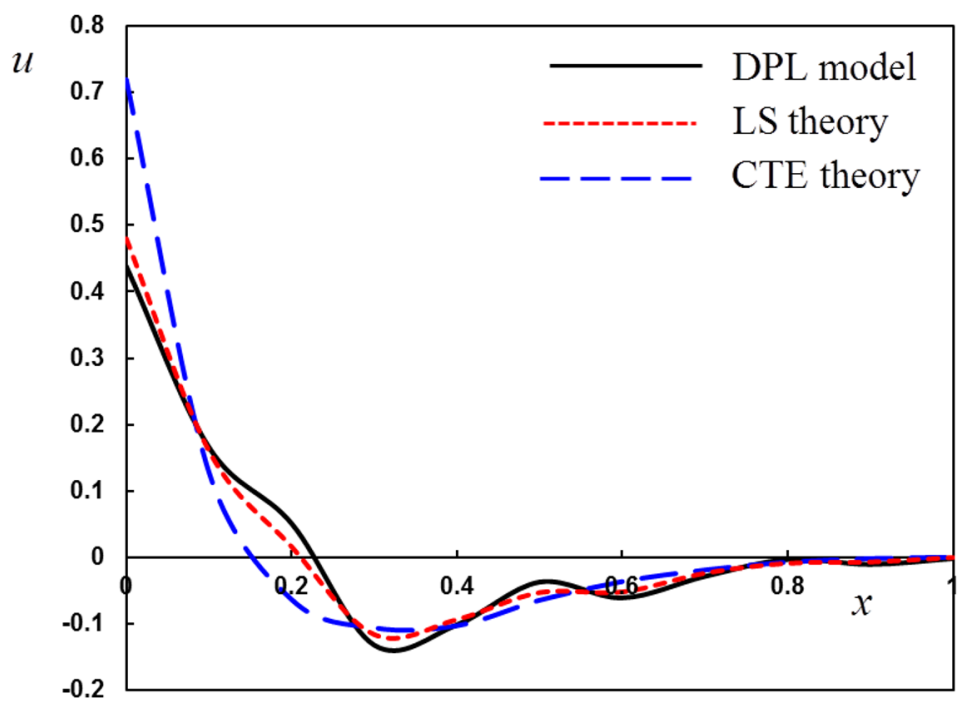

Figure 9: The displacement distribution $u$ with distance $x$ for different theories of thermoelasticity.

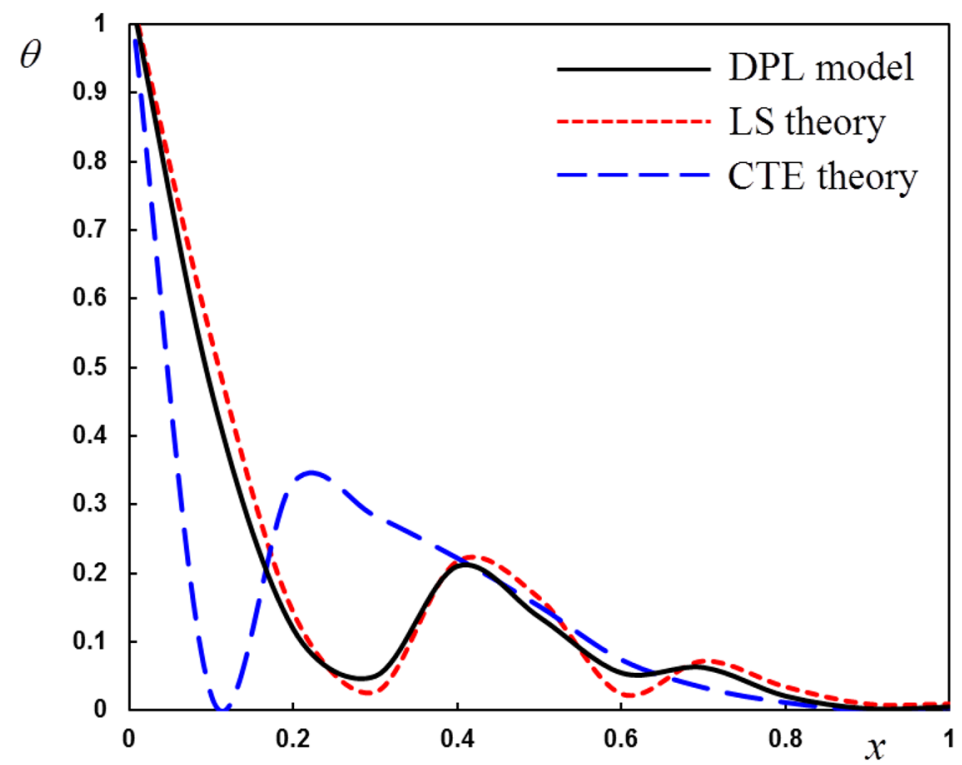

Figure 10: The thermodynamical temperature $\theta$ with distance $x$ for different theories of thermoelasticity. 


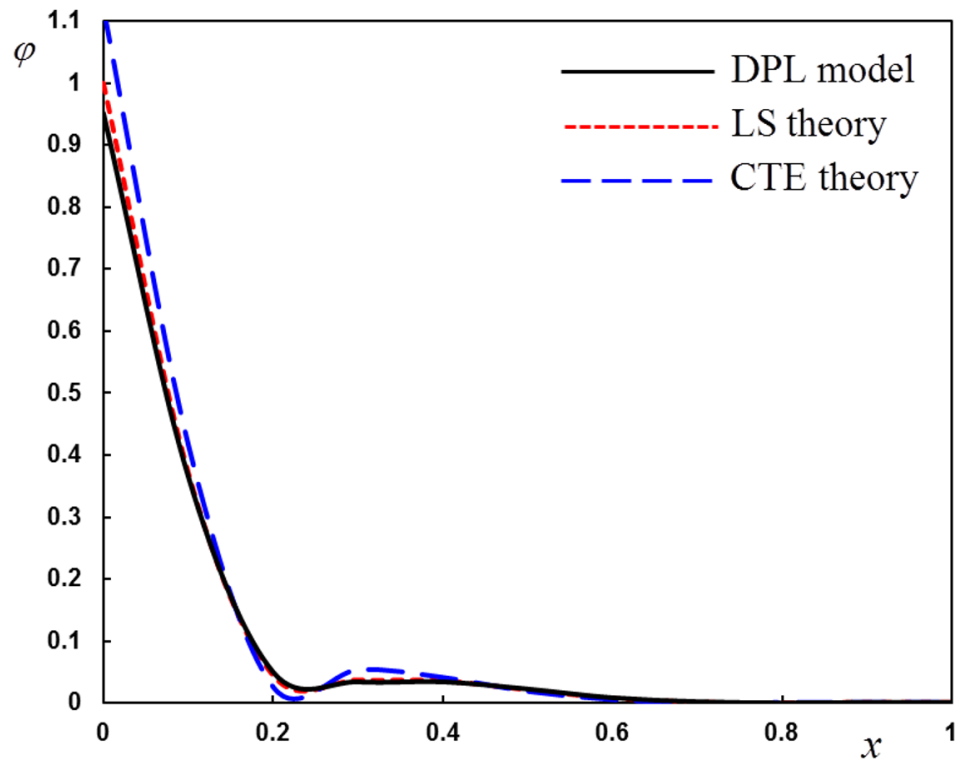

Figure 11: The conductive temperature $\varphi$ with distance $x$ for different theories of thermoelasticity.

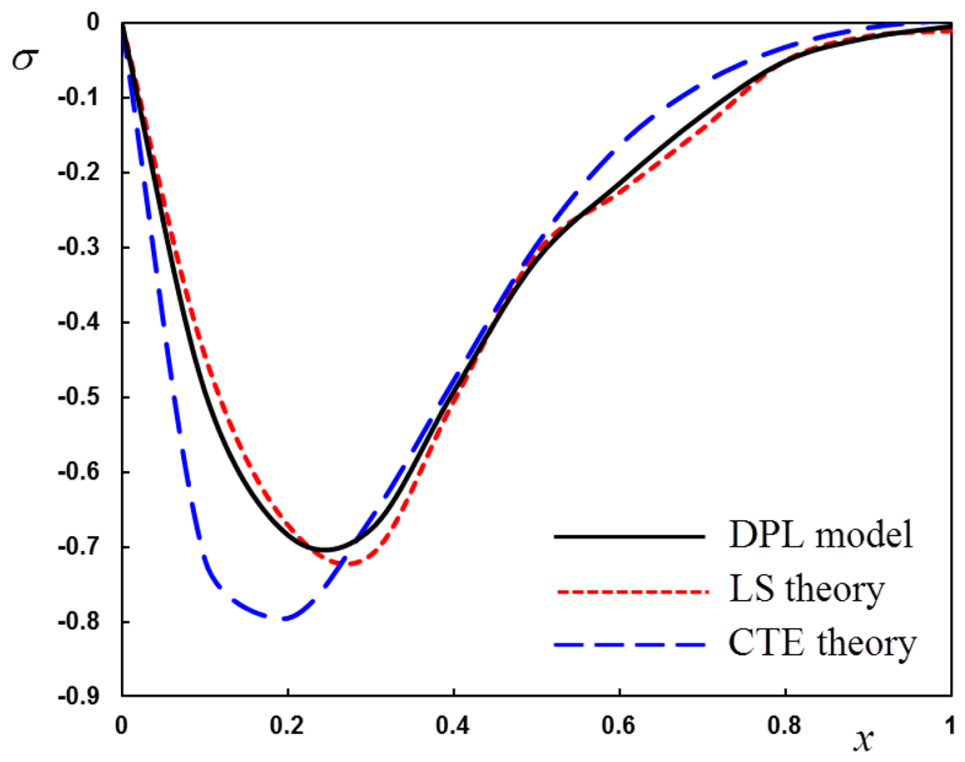

Figure 12: The thermal stress $\sigma$ with distance $x$ for different theories of thermoelasticity. 


\section{References}

[1] Biot M.A.: Thermoelasticity and irreversible thermodynamics. J. Appl. Phys. 27(1956), 3, 240-253.

[2] Lord H.W., Sulman Y.: A generalized dynamical theory of thermoelasticity. J. Mech. Phys. Solids 15(1967), 5, 299-307.

[3] Green A.E., Lindasy K.A.: Thermoelasticity. J. Elast. 2(1972), 1, 1-7.

[4] Tzou D.Y.: A unified field theory for heat conduction from macro- to micro- scale. ASME J. Heat Trans. 117(1995), 1, 8-16.

[5] Chen P.J., Gurtin M.E.: On a theory of heat conduction involving two temperatures. Z. Angew. Math. Phys. 19(1968), 4, 614-627.

[6] Chen P.J., Gurtin M.E., Williams W.O.: On the thermodynamics of non-simple elastic materials with two temperatures. Z. Angew. Math. Phys. 20(1969), 1, 107112 .

[7] Chen P.J., Gurtin M.E., Williams W.O.: A note on non simple heat conduction. Z. Angew. Math. Phys. 19(1968), 6, 960-970.

[8] Boley B.A., Tolins I.S.: Transient coupled thermoelastic boundary value problems in the half space. ASME J. Appl. Mech. 29(1962), 4, 637-646.

[9] Warren W.E., Chen P.J.: Wave propagation in the two temperature theory of thermoelasticity. Acta Mech. 16(1973), 1-2, 21-33.

[10] Youseff H.M.: The dependence of the modulus of elasticity and the thermal conductivity on the reference temperature in generalized thermoelasticity for an infinite material with a spherical cavity. J. Appl. Math. Mech. 26(2005), 4, 470-475.

[11] Wood R.F., White C.W., Young R.T.: Pulsed laser processing of semi- conductors. In: Semiconductors and Semimetals, Vol. 23, Chapt. 5, Academic Press, London 1984.

[12] Trajkowski D., CukiC R.: A coupled problem of thermoelastic vibrations of a circular plate with exact boundary conditions. Mech. Res. Commun. 26(1999), 2, $217-224$.

[13] Wang X., XU X.: Thermoelastic wave induced by pulsed laser heating. Appl. Phys. A, 73(2001), 1, 107-114.

[14] McDonald F.A.: On the precursor in laser-generated ultrasound waveforms in metals. Appl. Phys. Lett. 56(1990), 3, 230-232.

[15] Allam M.N.M., Aboulregal A.E.: The thermoelastic waves induced by pulsed laser and varying heat of non-homogeneous microscale beam resonators. J. Therm. Stresses 37(2014), 4, 455-470.

[16] Othman M.I.A., Tantawi R.S., ERAKi E.E.M.: Effect of rotation on a semiconducting medium with two-temperature under $L-S$ theory. Arch. Thermodyn. 38(2017), 2, 101-122.

[17] AbD-Alla A.N., Aво-Dahab S.M.: On the reflection of the generalized magnetothermo-viscoelastic plane waves. Chaos Soliton Fract. 16(2003), 2, 211-231. 
[18] AbD-Alla A.N., Abo-Dahab S.M.: The influence of the viscosity and the magnetic field on reflection and transmission of waves at interface between magnetoviscoelastic materials. Meccanica 43(2008), 5, 437-448.

[19] MukHopadhyay S.: Effects of thermal relaxations on thermoviscoelasticity interactions in an unbounded body with a spherical cavity subjected to a periodic loading on the boundary. J. Therm. Stresses 23(2000), 7, 675-684.

[20] Mukhopadhyay B., BerA R.K.: Effect of distributed instantaneous and continuous heat sources in an infinite conducting magneto-thermo-viscoelastic solid with thermal relaxation. Comput. Math. Appl. 18(1989), 8, 723-728.

[21] Ezat M.A., Othman M.I.A., El-Karamany A.S.: State-space approach to two dimensional generalized thermoviscoelasticity with two relaxation times. Int. J. Eng. Sci. 40(2002), 11, 1251-1274.

[22] Отhman M.I.A., Song Y.Q.: Effect of rotation on plane waves of the generalized electromagneto-thermo-viscoelasticity with two relaxation times. Appl. Math. Model. 32(2008), 5, 811-825.

[23] Othman M.I.A., FeKry M.: Effect of magnetic field on generalized thermoviscoelastic diffusion medium with voids. Int. J. Str. Stab. Dyn. 16(2016), 7 15500331-1550033-21.

[24] Tzou D.Y.: Macro- to microscale heat transfer: the lagging behavior. Series in chemical and mechanical engineering, Taylor \& Francis, Washington 1997.

[25] Sun Y., Fang D., Saka M., Soh A.K.: Laser-induced vibrations of micro-beams under different boundary conditions. Int. J. Solids Struct. 45(2008), 7-8, 1993-2013.

[26] Honig G., Hirdes U.: A method for the numerical inversion of the Laplace transform. J. Comput. Appl. Math. 10(1984), 1, 113-132. 